\title{
Asymptotic Analysis for Nonlinear Dynamic Problem of Functionally-Graded Shallow Shells with Time Dependent Thickness
}

\author{
Authors \\ V. Z. Gristchak ${ }^{1}$, Yu. A. Fatieieva ${ }^{2}$ \\ ${ }^{1}$ Asst.Professor of Mathematics Department, Zaporizhzhya National University, Zaporizhzhya, Ukraine \\ ${ }^{2}$ Postgraduate Student of Mathematics Department, Zaporizhzhya National University, Zaporizhzhya, \\ Ukraine \\ E-mail: fateevajulia@gmail.com
}

\begin{abstract}
The present paper deals with the problem of dynamic behavior of thin geometrically imperfect shallow shell structures made of functionally gradient material (FGM) with time dependent parameters. Special attention is paid to influence time dependent thickness and temperature effect. Hybrid asymptotic approach is used to obtain an approximate analytical solution of the problem. The material properties are graded in the thickness direction according to the given power law distribution. The non-linear strain-displacement relationships based upon the von Karman theory for moderately large normal deflections. Discussed problem leads to a singular nonlinear second order non homogeneous differential equation with variable in time coefficients. An analytical solution by hybrid perturbation-WKB-Galerkin $(P-W K B-G)$ method in some parameters of structure is compared with direct numerical integration results of initial problem equation.

Keywords: functionally graded material (FGM), hybrid asymptotic approach, approximate analytical solution, time dependent thickness.
\end{abstract}

\section{INTRODUCTION}

Thin walled structures made of functionally graded materials (FGM) with ceramic-metal, ceramicmetal-ceramic or a combination of other materials are widely used, for example, in aircraft and space systems due to their advantages of high heatresistance and stiffness. In recent years important studies have been researched about vibration and stability FGM plates and shells with using mostly by numerical approaches $[1-4,12]$. The present research devoted to analysis for nonlinear dynamics of imperfect FGM shell structures with variable in time thickness and influence of external dynamic and temperature loads on the basis of a hybrid asymptotic method. The main objective of the paper is to show that analytical approach on the basis of hybrid asymptotic methods can be effective for solution of nonlinear differential equations with variable coefficients.

\subsection{Basic Concept of the Hybrid Approach to Solution of Nonlinear Problems}

To solve the non-linear differential equations with variable in time coefficients the approach is applied in three stages:

1. the solution is determined using perturbation method by forming an expansion in parameter near the non-linear term of initial equation and we obtain the related system of linear non-homogeneous equations with variable coefficients;

2. the solutions of linear system are determined using the (one or two-step) WKBapproximation by forming an expansion in parameter;

3. the correction functions are obtained by classical Galerkin procedure. 


\subsection{The Nonlinear Dynamic Behavior of} Imperfect Shallow Shells with Variable in Time Thickness

An approximate analytical non-linear analysis is given on the basic system of equations in terms of the stress and deflection following to the paper [1]. Suppose the FGM shallow shell is simply supported at its edges and subjected to a transverse load $q_{0}(t)$ and compressive edge loads $r_{0}(t), p_{0}(t)$. We assume that modulus of elasticity and the mass density changes in the thickness direction, while the Poisson ratio is assumed to be constant and thickness of shell is function of time.

Appling Bubnov-Galerkin procedure with assumption that initial imperfection of middle surface of shell has the form:

$$
w_{0}\left(x_{1}, x_{2}\right)=f_{0} \sin \frac{m \pi x_{1}}{a} \sin \frac{n \pi x_{2}}{b},
$$

where $f_{0}$ is given amplitude, the non-linear second - order ordinary differential equation for function $f(t)$ with deflection function $w=\left(x_{1}, x_{2}, t\right)$ :

$$
w\left(x_{1}, x_{2}, t\right)=f(t) \sin \frac{m \pi x_{1}}{a} \sin \frac{n \pi x_{2}}{b},
$$

that are correspond to simply support boundary conditions, is given in the form [1]:

$$
\varepsilon^{2} \frac{d^{2} f}{d t^{2}}+B_{1}(t) f+\mu\left(B_{2}(t) f^{2}+B_{3}(t) f^{3}\right)=\bar{Q}_{0}(t)
$$

where

$$
\begin{aligned}
& \omega_{m n}^{2}=\frac{1}{\rho_{1}(t)}\left[\frac{\left(E_{1} E_{3}-E_{2}^{2}\right)}{E_{1}\left(1-v^{2}\right)} \cdot \frac{\left(m^{2}+n^{2} \lambda^{2}\right) \pi^{2}}{a^{4}}+\frac{E_{1}\left(k_{1} n^{2} \lambda^{2}+k_{2} m^{2}\right)^{2}}{\left(m^{2}+n^{2} \lambda^{2}\right)^{2}}\right] \\
& \bar{A}_{i}=\frac{A_{i}}{\omega_{m n}^{2}}, \varepsilon^{2}=\frac{1}{\omega_{m n}^{2}}, A_{1}(t)=\frac{\pi^{2} h(t)}{a^{2}}\left(m^{2} r_{0}+n^{2} \lambda^{2} p_{0}\right), \\
& A_{2}(t)=\frac{16 E_{1}(t) m n \lambda^{2}\left(k_{1} n^{2} \lambda^{2}+k_{2} m^{2}\right)}{3 a^{2}\left(m^{2}+n^{2} \lambda^{2}\right)^{2}}, \\
& E_{1}(t)=\left(E_{m}+\frac{E_{c}-E_{m}}{k+1}\right) h(t), \rho_{1}=\left(\rho_{m}+\frac{\rho_{c}-\rho_{m}}{k+1}\right) h(t) \\
& B_{1}(t)=1+2 a_{1}(t) m^{2} n^{2} \lambda^{4} \\
& \left.B_{2}(t)=\frac{-3}{\mu} \bar{A}_{2}(t)-\bar{A}_{3} \lambda^{2}\right)^{2}(t), B_{3} f_{0}^{2}-\bar{A}_{1}(t)=\frac{1}{\mu} \bar{A}_{3}(t), \\
& \bar{Q}_{0}(t)=Q_{0}-\bar{A}_{0}(t)+f_{0}-\bar{A}_{2}(t) f_{0}^{2} .
\end{aligned}
$$

$k_{1}, k_{2}$ - curvatures of middle surface shell in $x_{1}$ and $x_{2} ; \varepsilon, \mu$ are parameters.

Initial conditions for the equation (3) have form:

$$
\begin{aligned}
& \varphi(0)=1 \\
& \varphi^{\prime}(0)=0
\end{aligned}
$$

According to perturbation method and $[5,15]$ with respect to parameter of nonlinearity $\mu$, solution of differential equation (3) we obtain in the form of two terms approximation:

$$
\begin{aligned}
& f(t)=\phi_{0}(t)+\mu \phi_{1}(t)=B_{1}(t)^{0,25}\left[\operatorname { s i n } K ( t ) \left(c_{1}+\bar{c}_{1}(t)+,\right.\right. \\
& \left.\left.\mu \bar{d}_{1}(t)\right)+\cos K(t)\left(c_{2}+\bar{c}_{2}(t)+\mu \bar{c}_{2}(t)\right)\right]
\end{aligned}
$$

where

$$
\begin{gathered}
\bar{c}_{1}(t)=\varepsilon \int \frac{\bar{Q}_{0}(t) \cos K(t)}{B_{1}^{-0.25}(t)} d t, \bar{c}_{2}(t)=-\varepsilon \int \frac{\bar{Q}_{0}(t) \sin K(t)}{B_{1}^{-0.25}(t)} d t, \\
\bar{d}_{1}(t)=\varepsilon \int \frac{\left(-B_{2}(t) \varphi_{0}^{2}-B_{3}(t) \varphi_{0}^{3}\right) \bar{Q}_{0}(t) \cos K(t)}{B(t)^{-0.25}} d t \\
\bar{d}_{2}(t)=-\varepsilon \int \frac{\left(-B_{2}(t) \varphi_{0}^{2}-B_{3}(t) \varphi_{0}^{3}\right) \bar{Q}_{0}(t) \sin K(t)}{B_{1}(t)^{-0.25}} d t \\
K(t)=\int \varepsilon^{-1} B_{1}^{0.25}(t) d t
\end{gathered}
$$

constants $c_{1}, c_{2}, d_{1,}, d_{2}$ are obtained from initial condition (5).

For the given shell parameters numerical; results are presented in Fig.1-4.

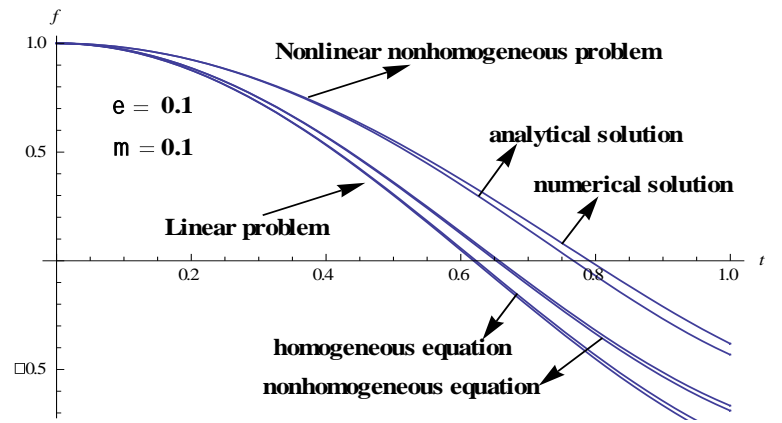

Fig. 1 Comparison of analytical and numerical solutions

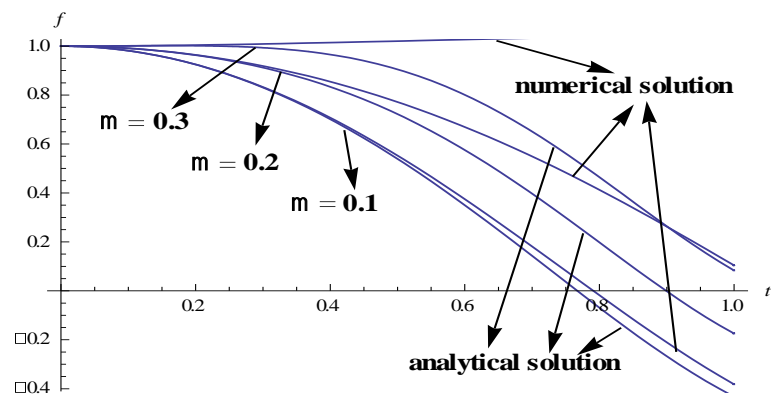

Fig. 2 Influence of nonlinear parameter $\mu$. (Comparison of analytical and numerical solutions at $\varepsilon=0.1)$ 


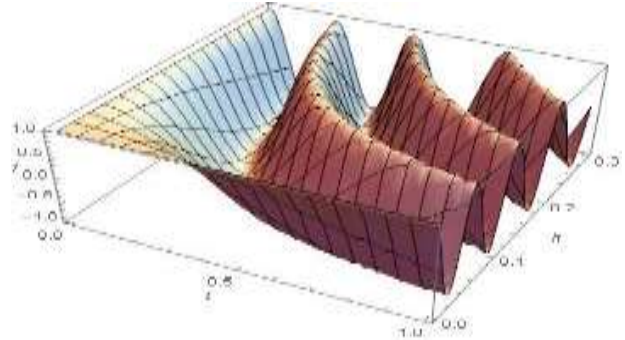

Fig. 3 Behavior of nonlinear dynamic process of imperfect shallow shell with variable thickness in time

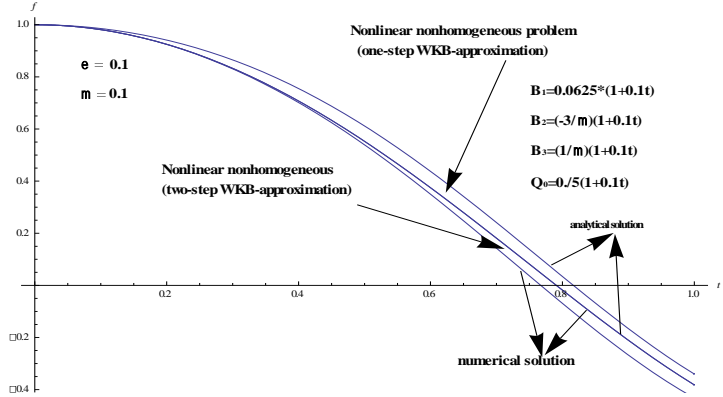

Fig. 4 Comparison of analytical and numerical solutions for nonhomogeneous linear and nonlinear problems (one-step and two-step WKBapproximations)

Influence of static loading and initial imperfection with respect to the following parameters of shallow shell are shown on the Fig. 5-6.

$h(t)=h_{0}(1-\eta t), Q_{0}=\operatorname{Sin} \Omega t, a=k_{1} p_{0}+k_{2} r_{0}$

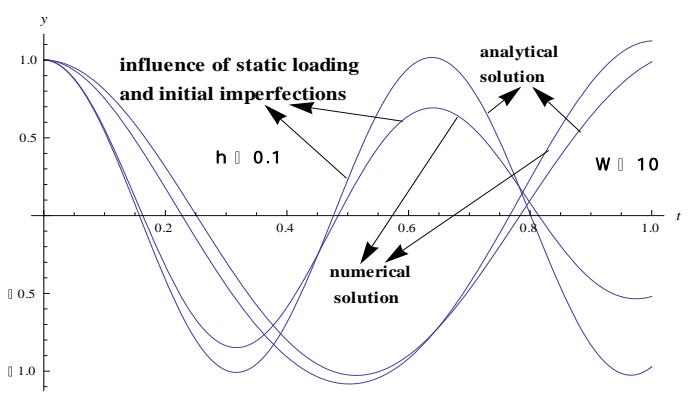

Fig. 5 Influence of static loading and initial imperfection

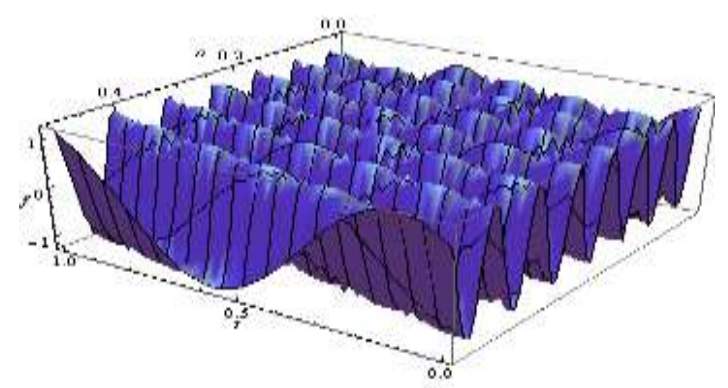

Fig. 6 Forced oscillations of imperfect shallow shells under static load
Dependence of shallow shell natural frequency vibration from index material graded is given on Table 1 and Fig. 7 - 8.

\begin{tabular}{|c|c|}
\hline $\begin{array}{c}\text { Index } \\
\text { gradation } \\
\text { of material } \\
k\end{array}$ & $\begin{array}{c}\text { Value of natural frequency of } \\
\text { material }\end{array}$ \\
\hline 0 & $w_{m n}^{2}=100,9 h^{2}(t) \cdot 10^{6}+4 \cdot 10^{6}$ \\
\hline 1 & $w_{m n}^{2}=64,83 h^{2}(t) \cdot 10^{6}+2,77 \cdot 10^{6}$ \\
\hline 2 & $w_{m n}^{2}=53,57 h^{2}(t) \cdot 10^{6}+2,25 \cdot 10^{6}$ \\
\hline 5 & $w_{m n}^{2}=48,69 h^{2}(t) \cdot 10^{6}+1,16 \cdot 10^{6}$ \\
\hline
\end{tabular}

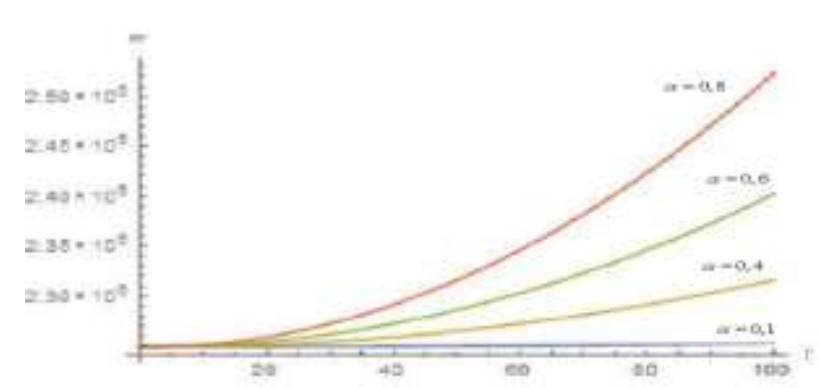

Fig. 7 The function of the natural oscillation frequency for changing the thickness $h(t)=h_{0}(1-\alpha t)$

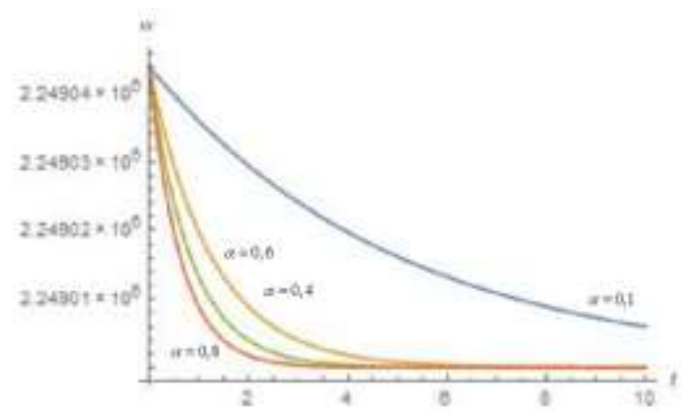

Fig. 8. The function of the natural oscillation frequency for changing the thickness $h(t)=h_{0} e^{-\alpha t}$

\section{ANALYTICAL SOLUTION FOR NONLINEAR THERMODYNAMIC PROBLEM OF FGM SHALLOW SHERICAL SHELLS WITH VARIABLE IN TIME THICKNESS}

The general material properties of FGM spherical shell with time dependent thickness, such as Young's modulus E(z), Poisson's ratio is constant $v(z)$, the mass density $\rho(z)$, coefficient of thermal expansion $a(z)$ coefficient of thermal conduction $K(z)$ and volume fraction are expressed in the form: 


$$
\begin{gathered}
E(z)=E_{m}+\left(E_{c}-E_{m}\right)\left(\frac{2 z+h(t)}{2 h(t)}\right)^{k}, \\
\rho(z)=\rho_{m}+\left(\rho_{c}-p_{m}\right)\left(\frac{2 z+h(t)}{2 h(t)}\right)^{k}, \\
\alpha(z)=\alpha_{m}+\left(\alpha_{c}-\alpha_{m}\right)\left(\frac{2 z+h(t)}{2 h(t)}\right)^{k}, \\
K(z)=K_{m}+\left(K_{c}-K_{m}\right)\left(\frac{2 z+h(t)}{2 h(t)}\right)^{k},
\end{gathered}
$$

The volume-fractions of the metal and ceramic phases base by power law $v_{m}+v_{c}=1,(m-$ belongs to metal, $c-$ to ceramic), $V_{c}=\left(\frac{2 z+h(t)}{2 h(t)}\right)^{k}$, where $k$ is the volume-fraction exponent $(k \geq 0)$ and $h(t)$ is time dependent thickness, ${ }^{r}$ is base radius, $R$ - curvature radius, $H$ is rise of shallow spherical shell.

An FGM shallow spherical shell defined in coordinate system $(\varphi, \theta, z)$, where $\varphi$ is in the meridian and $\theta$ is in circumferential directions of the shell and $z$ is perpendicular to the middle surface positive in ward. The radius of parallel circle of shell is $r=R \sin \varphi$ (Fig. 7).

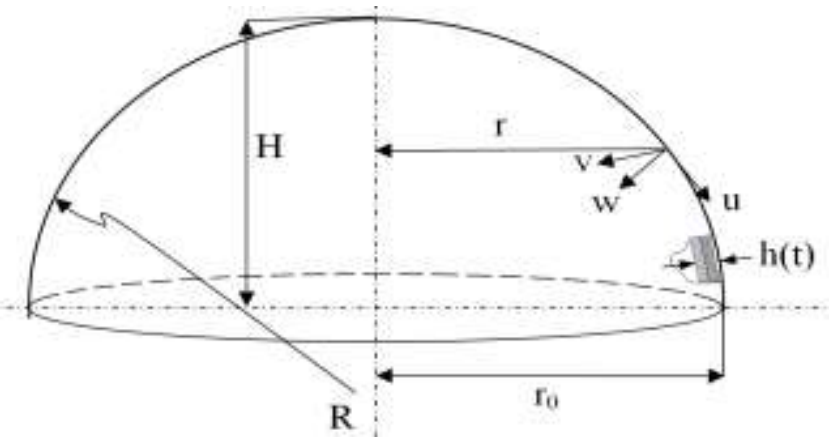

Fig. 9 Geometry and coordinate system of the functionally graded spherical shell with time dependent thickness

The governing equations used to investigate the nonlinear dynamic behavior of FGM shallow spherical shells with time dependent thickness according to [15] is:

$$
\rho^{*} h^{2} W_{, t t}^{*}+\frac{E_{1}^{*}}{\left(38-20 n^{2}+12 n^{4}\right)}\left\{\left[\frac{7 D^{*}\left(38-20 n^{2}+12 n^{4}\right)^{2}}{4 \xi^{4}}\left(\frac{h(t)}{R}\right)^{4}+\right.\right.
$$

$$
\begin{aligned}
& +\left(\frac{\left(3+2 n^{2}\right)^{2}}{7}+\frac{28\left(266-170 n^{2}+64 n^{4}\right)}{105 \pi^{2} n^{2}(1-v)}\right)\left(\frac{h(t)}{R}\right) \\
& -\left[\frac{96\left(3+2 n^{2}\right) n}{7 \pi \xi^{2}}+\frac{8\left(3+2 n^{2}\right)\left(266-170 n^{2}+64 n^{4}\right)}{105 \pi n(1-v) \xi^{2}}+\right. \\
& \left.+\frac{224\left[\pi^{2}\left(38-20 n^{2}+12 n^{4}\right)-40 n^{2}\right]}{105 \pi^{3} n(1-v) \xi^{2}}\right]\left(\frac{h(t)}{R}\right)^{3} W^{* 2}+
\end{aligned}
$$

$\left.+\left[\frac{2048 n^{2}}{7 \pi^{2} \xi^{4}}+\frac{64\left(3+2 n^{2}\right)\left[\pi^{2}\left(38-20 n^{2}+12 n^{4}\right)-40 n^{2}\right]}{105 \pi^{2} \xi^{4}(1-v)}\right]\left(\frac{h(t)}{R}\right)^{4} W^{* 3}\right\}-$ $-\frac{\varphi_{m}}{(1-v) h}\left[\frac{\left(3+2 n^{2}\right)}{2 \xi^{2}}\left(\frac{h(t)}{R}\right)^{2} W^{*}-\frac{7}{4 \pi n}\left(\frac{h(t)}{R}\right)\right]=\frac{7 q}{8 \pi n}$.

Environment temperature is uniformly raised from initial value $T_{i}$, at which the shell is thermal stress free, to final one $T_{f}$ and temperature change $\Delta T=T_{f}-T_{i}$ is dependent to thickness variable. The thermal parameter $\varphi_{m}$ can be expressed in terms of ${ }_{\Delta T}$ as $\varphi_{m}=\Phi_{m 0} \Delta T h(t)$,

where

$$
\Phi_{m 0}=E_{m} \alpha_{m}+\frac{E_{m} \alpha_{c m}+E_{c m} \alpha_{m}}{k+1}+\frac{E_{c m} \alpha_{c m}}{2 k+1} .
$$

Equation (11) is employed to obtain an approximate analytical solution of discussed nonlinear problem. The basic equation (11) can be rewrite as

$$
\begin{aligned}
& \rho^{*} h^{2}(t) \ddot{\tilde{w}}+e(t)\left\{\left[\bar{a}(t)+b_{\underline{1}}(t)\right] \tilde{w}(t)-\left[c_{t}(t)\right] \tilde{w}^{2}+\right. \\
& \left.+\left[d_{t}(t)\right] \tilde{w}^{3}\right\}-\frac{\varphi_{m}}{(1-v) h(t)}\left[\tilde{f}_{t}(t) \tilde{w}(t)-\tilde{g}_{t}(t)\right]=\frac{7 q}{8 \pi n},
\end{aligned}
$$

or

$$
\ddot{\tilde{w}}+A_{t}(t) \tilde{w}(t)=N_{t}(t)+Q_{t}(t)
$$

where

$$
\begin{gathered}
\overline{()}=\frac{()}{\rho h^{2}(t)}, \rho^{*}=\frac{\rho}{h(t)} \\
\rho_{1}=\rho_{m} \frac{h(t)}{k+1}+\rho_{m} h(t), \rho_{c m}=\rho_{c}-\rho_{m}, \zeta=\frac{r_{0}}{R} \\
\alpha_{c m}=\alpha_{c}-\alpha_{m}, \quad \rho^{*}=\frac{\rho_{m} k+\rho_{c}}{k+1} h(t)
\end{gathered}
$$




$$
\rho^{*}=\rho_{m} h(t)+\frac{\rho_{c}-\rho_{m}}{k+1} h(t)=\left[\rho_{m}+\frac{\rho_{c}-\rho_{m}}{k+1}\right] h(t) ; \quad \begin{aligned}
& \ddot{\tilde{w}}_{0}(t)+\alpha \ddot{\tilde{w}}(t)+A_{t}(t)\left[\tilde{w}_{0}(t)+\alpha \tilde{w}_{1}(t)\right]= \\
& =\alpha \tilde{N}_{t}(t)+Q_{t}(t)
\end{aligned}
$$

$D^{\prime \prime}(t)=\frac{D}{E_{1} h^{2}(t)}, \tilde{e}(t)=\frac{E_{1}^{\prime}(t) h(t)}{\left(38-20 n^{2}+12 n^{4}\right) \rho_{1}}$,

here $\alpha$ is "small" parameter and

$$
\begin{array}{r}
N_{t}(t)=\alpha \bar{N}_{t}(t), \\
\tilde{w}(t)=\tilde{w}_{0}(t)+\alpha \tilde{w}_{1}(t) .
\end{array}
$$

$b_{t}(t)=\left[\frac{\left(3+2 n^{2}\right)^{2}}{7}+\frac{28\left(266-170 n^{2}+64 n^{4}\right)}{105 \pi^{2} n^{2}(1-v)}\right]\left[\frac{h^{2}(t)}{R}\right]$ The hybrid asymptotic approach with respect to asymptotic) $\square$ and $\quad \square \square$ (singular $\quad$ internal $N_{t}(t)=\zeta_{t}(t) \tilde{w}(t)^{2}+D_{t}(t) \tilde{w}^{3}(t)$,

$\tilde{w}(t)=\frac{w(t)}{h_{0}} \quad, \quad a(t)=\frac{7 D\left(38-20 n^{2}+12 n^{4}\right)^{2}}{E_{1} h^{2}(t) 4 \xi^{4}}$ asymptotic) $\square$ leads to an approximate analytical solution [8] in the form

$A_{t}(t)=\tilde{e}(t)\left[a_{t}+b_{t}(t)\right]-\tilde{\varphi}_{m}(t)$,

$b_{t}(t)=\left[\frac{\left(3+2 n^{2}\right)^{2}}{7}+\frac{28\left(266-170 n^{2}+64 n^{4}\right)}{105 \pi^{2} n^{2}(1-v)}\right]\left[\frac{h^{2}(t)}{R}\right]$ $\tilde{w}(t)=\sin k(t)\left\{s_{1}+\left[\int \frac{Q_{t}(t) \cos k(t)}{k(t)}+\right.\right.$

$\left.\left.+\alpha \int \frac{e_{t}(t) \tilde{w}_{0}^{2}(t)+D_{t}(t) w_{0}^{3}(t)}{k(t)} \cos k(t) d t\right]\right\}+$ $+\cos k(t)\left\{s_{2}-\left[\int \frac{Q_{t}(t) \sin k(t)}{k(t)} d t-\right.\right.$,

$\left.c_{t}(t)=\left[\frac{96\left(3+2 n^{2}\right) n}{7 \pi \xi^{2}}+\frac{8\left(3+2 n^{2}\right)\left(266+170 n^{2}+64 n^{4}\right)}{105 \pi n(1-v) \xi^{2}}+{ }_{-\alpha} \int \frac{e_{t}(t) \tilde{w}_{0}^{2}(t)+D_{t}(t) w_{0}^{3}(t)}{k(t)} \cdot \sin k(t) d t\right]\right\}$.

$$
\left.+\frac{224\left(\pi^{2}\left(38-20 n^{2}+12 n^{4}\right)-40 n^{2}\right)}{105 \pi^{2} n(1-v) \xi^{2}}\right]\left[\frac{h(t)}{R}\right]^{3}, \quad \begin{aligned}
& \text { where } \\
& k(t)=\int \varepsilon^{-1} \bar{A}_{t}(t) d t, \varepsilon^{2}=\xi^{4}, \\
& \xi=0.2
\end{aligned},
$$

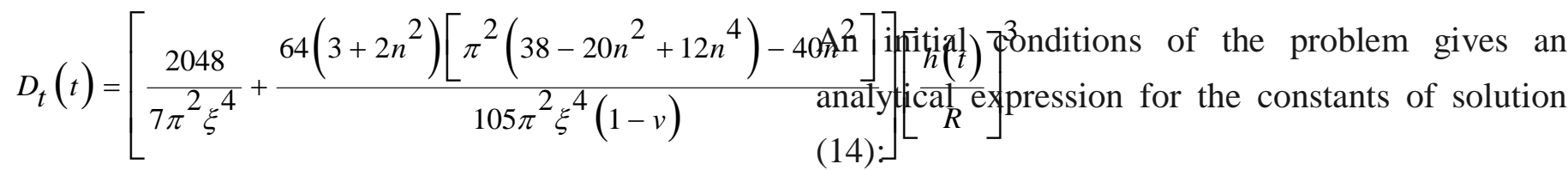
$\tilde{\varphi}_{m}(t)=\frac{\varphi_{m}}{(1-v) h(t)}\left[\frac{\left(3+2 n^{2}\right)}{2 \xi^{2}}\left[\frac{h(t)}{R}\right]^{2}\right]$,

$$
\begin{gathered}
s_{1}=\frac{1}{2} \sin 2 k(0)\left\{1-\tan k(0) \Omega_{1}(0)\right\}+ \\
+\frac{1}{\dot{k}(0)} \Omega_{1}(0)-\cos ^{2} k(0) \Omega_{1}(0)-\frac{1}{\dot{k}(0)} \dot{\Omega}_{2}(0),
\end{gathered}
$$$$
Q_{t}(t)=\frac{7 q(t)}{8 \pi n \rho_{1} h(t)}-\frac{\tilde{\varphi}_{m}}{(1-v) h(t)} \cdot \frac{7}{4 \pi n}\left[\frac{h(t)}{R}\right] \text {, }
$$

$f_{t}(t)=\frac{3+2 n^{2}}{2 \xi^{2}} \cdot \frac{1}{\rho_{1} h(t)}, \quad g_{t}(t)=\frac{7}{4 \pi n} \cdot \frac{1}{\rho_{1} h(t)} ;$

$$
\begin{gathered}
s_{2}=\left[1-\operatorname{Tan} k(0) \Omega_{1}(0)-\Omega_{2}(0)\right]- \\
-\sin ^{2} k(0)\left[1-\tan k(0) \Omega_{1}(0)\right]-
\end{gathered}
$$

with initial conditions

$$
\begin{aligned}
& \left.\frac{d \tilde{w}(t)}{d t}\right|_{t=0}=0, \\
& \tilde{w}_{0}=1 .
\end{aligned}
$$

Basic equation of the problem is introduced as

$$
\begin{aligned}
& -\frac{\tan k(0)}{\dot{k}(0)} \dot{\Omega}_{1}(0)-\frac{1}{2} \sin 2 k(0) \Omega_{1}(0)- \\
& -\frac{1}{2} \sin ^{2} k(0) \Omega_{1}(0)-\frac{\tan k(0)}{\dot{k}(0)} \dot{\Omega}_{2}(0)
\end{aligned}
$$


Comparisons of analytical and numerical solutions with influence of temperature are given on Fig. 1012.

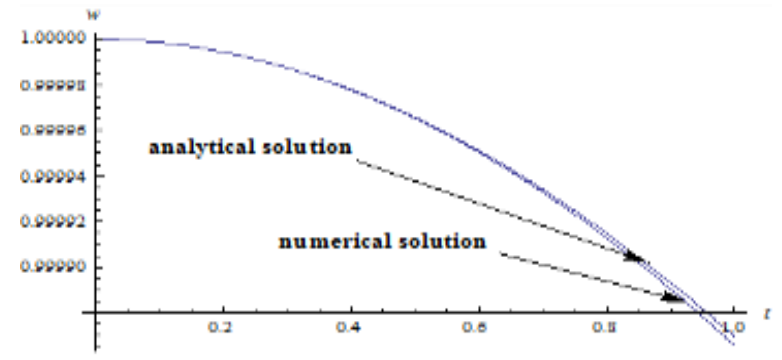

Fig. 10 Free vibrations with influence of temperature

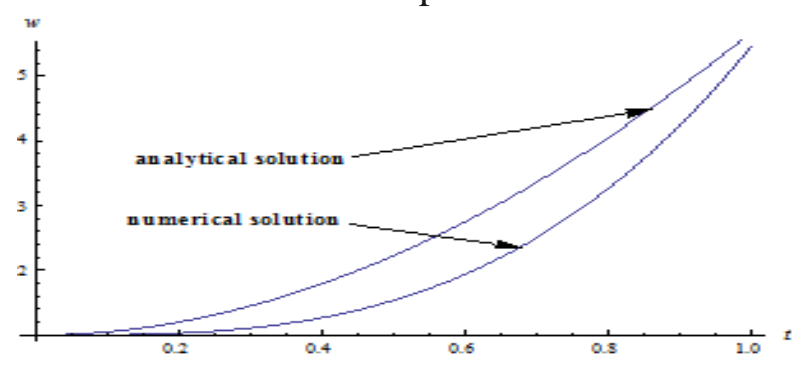

Fig. 11 Forced vibrations with influence of temperature

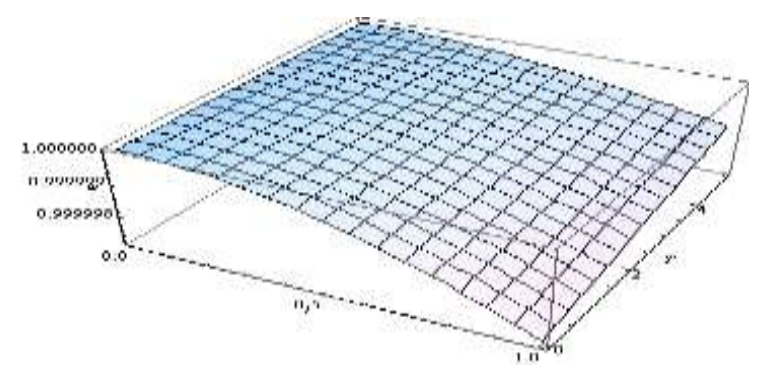

Fig. 12 Dependence the amplitude of the shell from the parameter of temperature loading

\section{CONCLUSIONS}

An approximate analytical solution for forced oscillations of geometrically non-linear FGM imperfect shallow cylindrical shells with time dependent parameters including temperature effects on the basis of hybrid perturbation-two-terms WKB approximation method are obtained. Some numerical calculations for the shell with variable in time parameters and comparison of approximate analytical solutions with direct numerical integration of initial nonlinear non homogeneous equation with variable coefficients are given. For particular parameters of structure an analytical solutions are in a good enough correlations with direct numerical solutions of initial singular nonlinear differential equations with variable in time coefficients. In some cases one-term WKBapproximation gives good enough results for the practical purpose.

\section{REFERENCES}

1. Dao Huy Bich, Yu Do Long: Nonlinear Dynamical Analysis of Imperfect Functionally Graded Materials Shallow Shells. Vietnam Journal og Mechanics, VAST, 32, 1 (2010), 1-14.

2. Vu Thi Thuy Anh, Nguyen Dinh Duc: Nonlinear Axisymmetric Response of Thin FGM Shallow Spherical Shells with Ceramic-Metal-Ceramic Layers under External Pressure and Temperature. VNU Journal of Mathematics - physics, 29, 2 (2013), 1-15.

3. Katarzhyna Kowal-Michalska, Radoslaw J. Mania: Static and Dynamic Thermomechanical Buckling Load of Functionally Graded Plate. Mechanics and Mechanical Engineering, 17, 1 (2013), 99112.

4. Khaire P.D., Ambhore N.H., Jagtap K.R.: Nonlinear Free Vibration Response of FGM Cylindrical Shell in Thermal Environment. Int. Journal on Mech. Engineering and Robotics, 2 (2014).

5. Geer, J.F., Andersen, C.V.: Natural Frequency Calculations Using a Hybrid Perturbation Galerkin Technique. Pan American Congress on Appl. Mech. (1991), 571-574.

6. Gristchak, V., Dmitrijeva, Ye.: A Hybrid WKB-Galerkin Method and its Application. Technische Mechanik, 15, 4, (1995), 281294.

7. Gristchak, V., Kabak, V.: Double Asymptotic Method for Nonlinear Forced Oscillations Problem of Mechanical Systems with Time Dependent Parameters. Technische Mechanik, 4, (1996), 285-296.

8. Gristchak V.Z., Ganilova O.A.: Application of a Hybrid WKB-Galerkin Methodin Control of the Dynamic Instability of a 
Piezolaminated Imperfect Column.

Technische Mechanik, 26, 2,(2006), 106-116.

9. Gristchak V.Z., Ganilova O.A.:

Tothedynamic deformation problem of piezoelectric sandwich plates on the basic of hybrid WKB-Galerkin method. Reportsof National Academy of Science of Ukraine, 5, (2008), 13-20.

10. Gristchak V.Z.; Ganilova O.A.: A Hybrid WKB-Galerkin Method Applied to a Piezoelectric Sandwich Plate Vibration Problem Considering Shear Force Effects. Journal of Sound and Vibration, 317, 1-2, (2008), 366-377.

11. Gristchak V.Z., Ganilova O.A.: Application of a Hybrid WKB- GalerkinMethodtoa Nonlinear Plate Dynamic Problem with Time Dependent Damping Coefficient. Proc. VI Int. Conf. EUROMECH ENOC, 80Min / 700MB, Sankt-Petersburg(2008).

12. Cartmell M.P.; Ganilova O.A.; Gristchak, V.Z.:

ControllingPlateVibrationsbyMeansofEmbe ddedActiveShapeMemoryAlloyElements.Eu romech 498 Colloquium: international conference: Proceedings of Euromech 498 Colloquium,498, (2008), 112-118.

13. Kurpa,L.V., Osetrov, A.A.; Shmatko, T.V.: Determination of eigen frequencies offunctionally graded material shallow shells with using of R-function and splineapproximation. Announcer of NTU CHPI, 6, (2014), 99-111.

14. Stephen Wolfram: Mathematica. A System for Doing Mathematica by the Computer. Wolfram Research Inc. (1988).

15. Gristchak V.Z., Fatieieva Yu.A.: An approximate nonlinear dynamic problem solution of functionally graded material shallow shell structure with in time thickness variation. Announcer of $Z N U, 3$, (2014). 